\title{
USER EXPERIENCE OPTIMIZATION USING PSYCHOPHISIOLOGICAL MEASURES
}

\author{
Martin ČERTICKÝ, Peter SINČÁK \\ Department of Cybernetics and Artificial Intelligence, Faculty of Electrical Engineering and Informatics, \\ Technical University of Košice, Letná 9, 04200 Košice, Slovak Republic, \\ Tel.: +421 55602 5165, E-mail: martin.certicky@tuke.sk, peter.sincak@tuke.sk
}

\begin{abstract}
With the increase in popularity of electronic entertainment (EE), the demand of optimization of its individual element arose. Big studios producing products of EE (movies, video-games, music, etc.) are benefiting from any gathered feedback from their customers. Customer's reaction to individual parts of the video-game might be invaluable for future level-designers, in form of information about each single aspect/feature targeted in the optimization process. In this paper, we present a study of individual checkpoints on the way of optimizing such EE products using artificial intelligence. We also present a way of using psychophysiological measurements of subjects playing video-games or watching movies to optimize their general EE experience.
\end{abstract}

Keywords: electronic entertainment, psychophysiological measurements, heart rate, video-games

\section{INTRODUCTION}

Psychophysiological measurements proved to be an objective, continuous, real-time, non-invasive and precise way to assess the game experience. Although for best results, it is needed to carefully control the experiment using the specialized equipment (both problems addressed in following sections).

Using the psychophysiological data, we should be able to find correlation between various features in tested domain and current phychophysiological state of the subject. Thus giving developer the information about how subjects reacts to current segment in the domain. In the past, researchers were trying to determine the players experience through set state, such as boredom, using known emotional states instead of psychophysiological measurements [7]. For example, if the subject playing a video-game is getting bored (state determined by an exact combination of psychophysiological states) while walking in the long corridor with no enemies, the level-designer has an option to add some enemies to the game (if the story line allows it - [12]). Some of the another options are to change the current music played, even changing the shape of the corridor completely using a Procedurally Generated Content (PCG) algorithm [4]. This implies that video-games may definitely be considered as an emotional experience [10].

There are numerous psychophysiological measurements which may be useful for this research: Heart Rate (HR), Electro-dermal Activity - Skin Conductivity (EDA), Facial Electromyography (EMG), Electrocardiogram (ECG), etc. [1]. Although so far we have only used HR data. In the future, we are planning to gather information about subject's EDA and their individual emotional states using image recognition [3]. This opens another interesting course of research - finding a correlation between psychophysiological measurements and emotional states of the subjects. Fleming an Rickwood studied the difference between violent and non-violent video-games on children's arousal, aggressive and positive moods [9].

In our research, we plan to incorporate continuous HR data into similar demo as shown on Fig. 2. We will try to find a correlation between individual game events and responses of the subjects. Afterwards, we plan to use artificial intelligence to optimize user experience in specific domain.

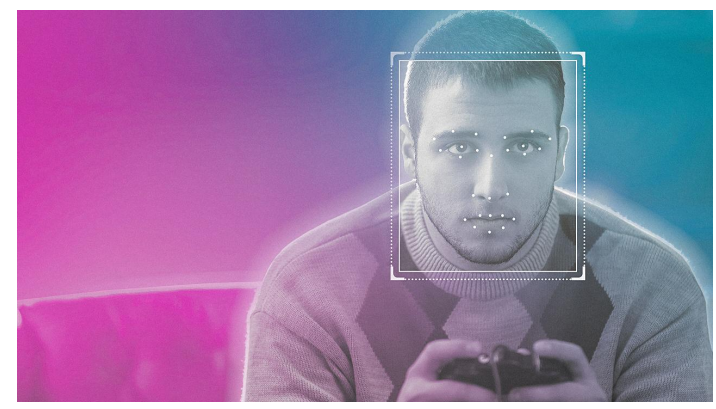

Fig. 1 What Happens When Video-Games Can Read Your Face [3]

\section{RELATED WORK}

Past research of psychophysiological responses may have been focused on a different goal then optimization EE products - such as work of Ballard an Wiest in 1996, where they studied the effects of violent video-games on male's hostility and their cardiovascular responses [5]. Another interesting feature (while very easy to control during the game) is an amount of blood in the game.

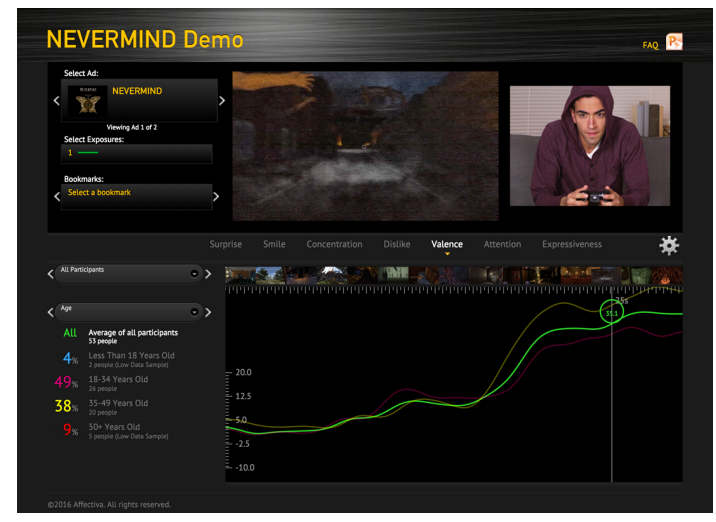

Fig. 2 Demo of Affectiva Software [15] 
Barlett et. al. studied the effect of the amount of blood in a violent video-game on aggression, hostility and arousal of the players [6].

Of course, the domain with highest psychophysiological changes of subjects are horror movies and video-games. Dekker and Champion focused on said domain studying the effect of fearful experiences on the subjects [8].

The experiments require fairly big number of subjects, since there are very different results throughout individuals as a players. Kallio et. al. determined up to 9 different types of gaming mentalities [11]. As stated in [13] by Tafalla, even subject's gender plays fairly important part in their psychophysiological responses during the expe iment.

There is a fairly similar research done at MIT, studying player's responses to video-game in real-time, using the data gathered from subject's face (see Fig. 1) using image recognition techniques [15]. We expect this research's results to be very useful in conjunction with our data, hopefully even finding correlation between them.

\section{MOTIVATION}

Most of the successful game-development companies are investing a significant amount of money in analytics departments in order to improve player experience. Also movie-making industry is using test screenings and focus groups to predict audience reaction before releasing their titles. Any information helping to achieve this goal is valuable to these departments even when not used right away. Therefore we expect this research to be helpful especially since there is currently no known dataset of psychophysiological measurements of players during gameplay.

\subsection{Movies}

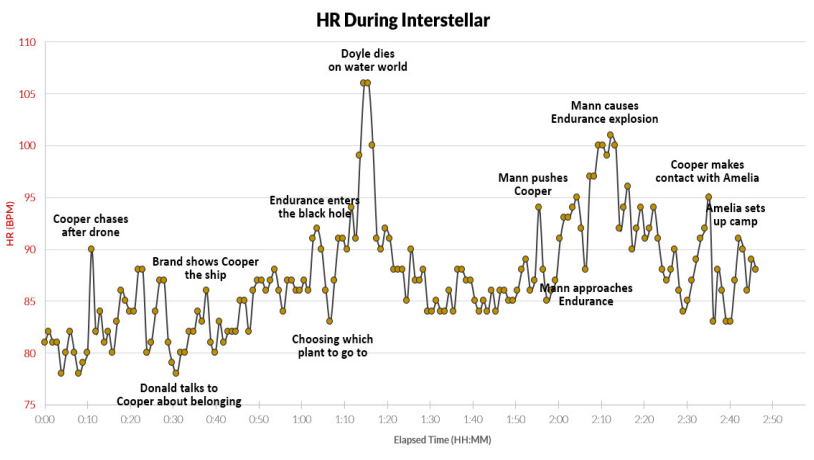

Fig. 3 HR of a subject watching the Interstellar movie [19]

Movies are fairly useful field to measure over-all userexperience of subjects, due to their static part in the process. While sitting still, there is a little chance of subject being affected by his/hers performance, communication with teammates etc. Even though the psychophysiological measurements may vary in regard of person's character and movie genre, the patterns in HR during the movies are still similar [18]. There is no logical point in having the viewer be as engaged as possible during the whole movie. Such experience may be exhausting, resulting in viewer being drained by the end of the movie. Most movies are aiming for up-and-down experience with exciting ending of the experience. For example, Fig. 3 shows HR of a subject while watching Interstellar with most interesting events in the movies denoted. This shows that the HR measurements should be greatly useful for movie industry.

\subsection{Single-player games}

Player engagement in single-player games should be very useful during the testing phase of the game. Similar to movies, single-player games are designed (with exceptions) to have the same story-lines or events to happen even when played repeatedly. When playing without outside distractions, player's heart rate should show any exciting/interesting parts of the game and more importantly, show any dull/uninteresting portions of the game.

The experiments regarding single-player games and movies are yet to be done.

\subsection{Multi-player games}

Unlike the single-player titles, multiple instances of multi-player games are rarely similar and are affected almost exclusively by player's actions. Because of this, the group of players joined together in multi-player game should meet certain characteristics in order to them enjoying the game as much as possible. The most important balancing characteristic is the skill level of players. Matching groups of players with different skill levels often results in none of the players enjoying the game. Even the players with the higher skill level do not feel satisfied when the game is not challenging enough. Therefore most of the significant multi-player titles are using sophisticated matchmaking systems trying to pick out the best combination of players possible for each game [20]. Matchmaking systems are generally using several KPI (Key Performance Indicators):

1. MMR (Matchmaking Rating) delta

2. Uncertainty delta (uncertainty rises with player not being consistent with his performances)

3. Party size

4. MMR spread of the players

5. Latency of the player

6. Time spent waiting for the game

7. Etc.

Naturally, particular KPIs for each game are different, but every matchmaking system is designed to maximize player enjoyment. Whether the player is or is not engaged in the game may be measured by his psychophysiological state. Such measurements can be invaluable for researchers working on matchmaking systems.

Even though we have some experimental results to work with already, most of the informations used by matchmaking systems are not public. Therefore, we are unable to find correlations with matchmaking KPIs and player engagement yet, even though it should be fairly obvious that the two are consequential. An example of applying our find- 
ings in practice could be based on one of our first findings: During the game of Dota 2, the biggest spike in subject's HR happens usually in last few minutes of the game, most likely during the game-deciding massfight (big encounter where most of the players are fighting enemy's team). In theory, this can tell the game developers that the shorter the game of Dota 2 is, the more frequently should subjects experience this event.

\section{SENSORS}

With the recent popularity increase of various wearable sensors, it has become easier for developers to get valuable psychophysiological data as feedback from the customer during his EE experience. Up to recently, the most used way were simple questionnaires presented to the customers or effort to measure emotions of the subject using various image recognition techniques.

As previously stated, we have been using only HR data of the subject so far. Also only one subject has been tested during gameplay. For gathering the HR data series, we have been using the Fitbit Surge HR ${ }^{1}$ sensor. After a brief survey of available wearable sensors, this one was chosen due to its ability to track HR during the whole day (instead of most available sensors which are tracking HR only during workout) and Fitbit's API provided for independent developers. The second sensor available for our research to gather HR data is Microsoft Band 2 which also meets said requirements 2

For gathering EDA data (specifically Galvanic Skin Response - GSR) we are planning to use either noncommercial sensors connected to Arduino/Raspberry Pi or Shimmer3 GSR unit, which is considerably more expensive. After gathering enough GSR data, we are planning to compare it with HR data and find any possible correlation (indulging the research done in [2]).

Lastly, we will be using the facial features extracted from video stream of subject using Microsoft Kinect v2 sensor. Although Microsoft's SDK for Kinect only provides 3 basic emotional states of the subject, using image recognition we might be able to gather more specific information about subject's mood (such as [15]).

List of sensors which will be used in the research and their potential (desired) application:

1. Fitbit Surge HR for obtaining Heart Rate data.

2. Microsoft Kinect v2 for getting emotion data from face features.

3. EDA sensor for obtaining Galvanic Skin Response data real-time, to compare with HR.

\section{PROBLEM DOMAINS}

Based on our extensive experience in the field, we have decided to try video-games as a starting domain of our research. Individual events in video-games are easy enough

\footnotetext{
${ }^{1}$ https://www.fitbit.com

${ }^{2}$ https://www.microsoft.com/microsoft-band

${ }^{3}$ http://blizzard.com

${ }^{4}$ http://www.valvesoftware.com/
}

to keep track on, which is a major advantage when finding a correlation between them and subject's HR. We have chosen two games: Dota 2 (see section 5.1) and Hearthstone: Heroes of Warcraft (see section 5.2), both among the most popular games in their fields.

\subsection{Dota 2}

Dota 2 is a multi-player online battle arena (MOBA) game set in a three-dimensional (3D) graphical environment, presented from an oblique high-angle perspective. Two five-player teams compete in matches on an asymmetrical playing field. Each player commands one of 111 "Hero" characters, which feature unique abilities and styles of play. At the start of a match, all Heroes have an experience level of one: they level up, and become more powerful, by accumulating experience points through combat. Whenever a Hero gains a level, the player may unlock a new ability for them, or enhance their statistics.

Considering that Dota 2 as a multi-player game is played by two teams of 5 players, the possibility of another interesting research arises: Studying the effects of game events on each player within the team as well as wholeteam impact. Having such data might open a possibility to simulate collaborative behavior of intelligent agents in a new way [16].

\subsection{Hearthstone: Heroes of Warcraft}

Hearthstone is a digital collectible card game that revolves around turn-based matches between two opponents, operated through Blizzard's ${ }^{3}$ Battle.net. Players can choose from a number of game modes, with each offering a slightly different experience.

Each Hearthstone match is a one-versus-one battle between two opponents. Gameplay in Hearthstone is turnbased, with players taking turns to play cards from their hand, casting spells, equipping weapons, or summoning 'minions' to do battle on their behalf. Unlike card games like Magic: The Gathering, the opposing player has no means to interactively interrupt or counter the current player's action during their turns, though may play cards on their turn that will create events that automatically respond to the other player's actions. Games may be between two players, or one human player and one computer-controlled opponent [17].

\section{EXPERIMENTS}

As previously stated, we already did some elementary experiments in our research. We have tracked the subject playing 2 different video-games - Valve's ${ }^{4}$ Dota 2 and Blizzard's Hearthstone: Heroes of Warcraft (Hearthstone). The games were played in carefully controlled environment, where subject was not disturbed by any outside events.

For the first round of experiments, we focused on gathering HR data of the subject using Fitbit Surge HR sensor (section 4). 
The subject was wearing Fitbit Surge HR sensor during the experiments with Fitbit App for Android communicating with the device (Fig. 4). For streaming (visual) purposes, the Android screen was mirrored and the portion of it showing live HR data was shown on Twitch.tv 5 stream, along with the current time and the video feed of the subject. After Fitbit Surge HR syncs with its cloud server, we were able to download any portion of recorded data with different detail-level (1 second, 1 minute or 15 minutes) we used 1 minute in our experiments.

These experiments already showed very different results emerging from the nature of the game itself. While Dota 2 as a Multi-player Online Battle Arena (MOBA) is more action-based game, Hearthstone is an turn-based card game.

Also, after a few sessions, we noticed the results vary slightly in each session. This might be caused by even minor changes in subject's current psychological or physiological state. Some of the factors affecting human involuntary neural system - thus the values of subject's HR are air temperature, body position, body size, medication use, even subject's satiation may take major part in the final outcome of the experiment.

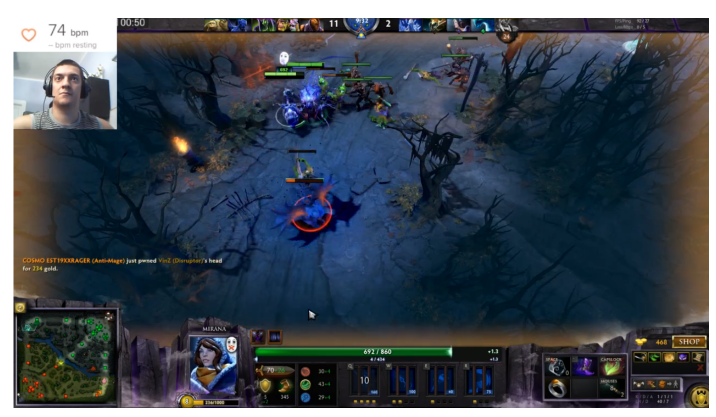

Fig. 4 Screenshot of subject during the game of Dota 2

This again emphasize the importance of careful control over the experiments during our future research. Another solution to this is to ignore absolute values of measured data and take only its deviations into consideration, similar to [14].

\subsection{Dota 2}

After 18 games of Dota 2 of which 9 has been won, the subject showed an average $\sim 83 \mathrm{HR}$ (see Fig. 5). Subject averaged $\sim 84$ HR during the winning games and $\sim 81$ average HR while losing. With bigger sample size, we should be able to detemine whether the losing/winning the game does have impact on subject's HR.

Resulting from the previous section, it has to be taken into consideration that recreating the same conditions for all experiments is near-to-impossible task. Especially when experiments are done with longer games, such as Dota 2, since it is difficult to play several games in a row at the same day.

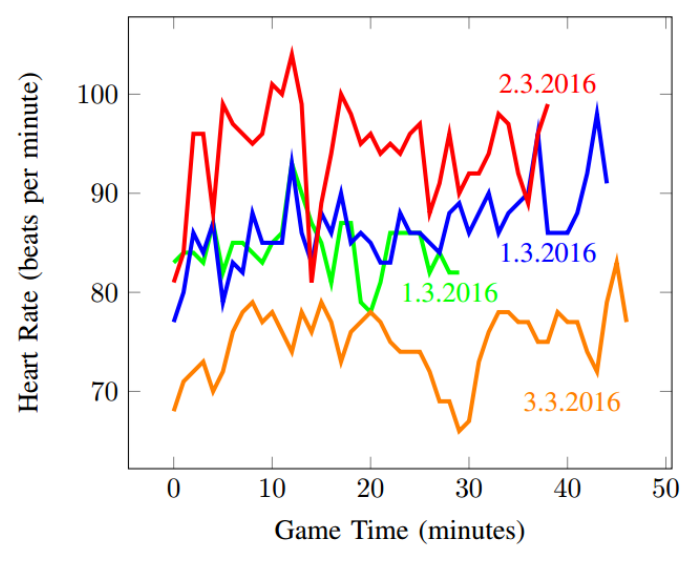

Fig. 54 games of Dota 2 played by tested subject in 3 sessions

Fig. 5 display similarities of subjects HR during 16 games of Dota 2, even though games were played in several different sessions. There are noticeable spikes of HR in several game stages, closely described in section 7

After only a small amount of games played, we were already able to find some interesting patterns emerging from our experiments. There are several spikes in the typical game of Dota 2, where the player is participating in socalled massfights. It is obvious that HR of the player is rapidly decreasing while the player is dead (waiting for respawn).

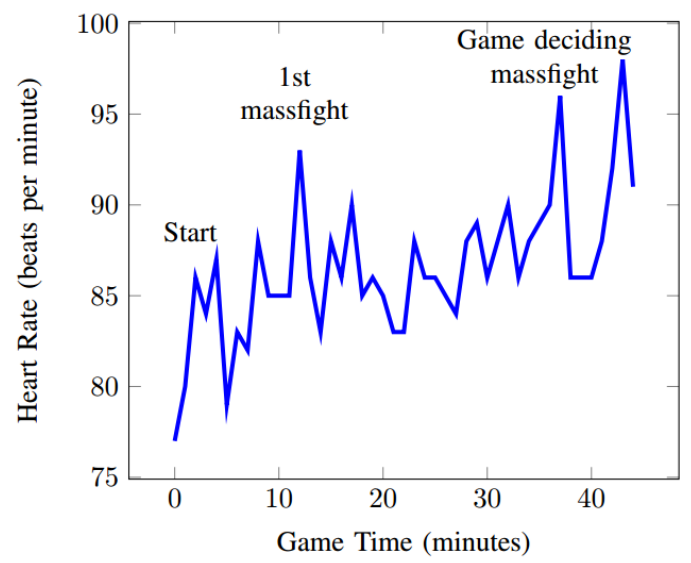

Fig. 6 Typical game of Dota 2 played by tested subject

Fig. 6 shows HR of subject during the game of Dota 2 . Subject's HR increases significantly during massfights (big encounter where most of the players are fighting enemy's team), while decreases when the player is dead (waiting to respawn).

In almost every game, there is also a significant spike in HR of player at the end of the game (see Fig. 6). This is caused due to most of the games ends with on plast attempt of losing team to defend its base before conceding the victory to the enemy. 


\subsection{Hearthstone: Heroes of Warcraft}

The subject played 3 sessions of Hearthstone games, one consisting of 4 games, other two of 2 games. Subject's average HR during the first two sessions was 76.97 (see Fig. 7).

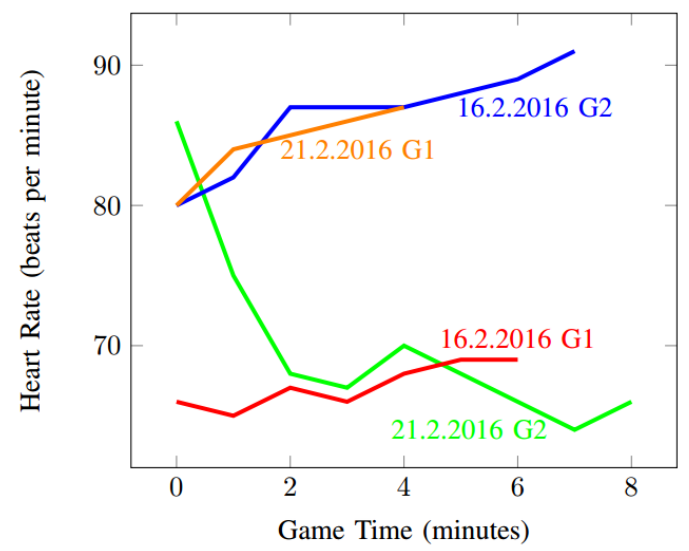

Fig. 7 Hearthstone: Heroes of Warcraft sessions 1+2

The third session showed average HR of 82,69 (see Fig. $8)$.

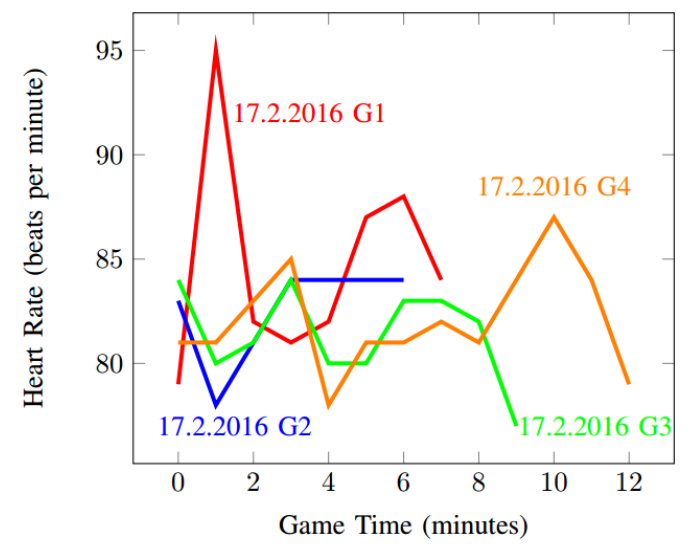

Fig. 8 Hearthstone: Heroes of Warcraft session 3

Both Fig. 7 and Fig. 8 show subjects HR during different Hearthstone games, played in different sessions. There are no obvious patterns to be seen due to small number of experiments done, this is to be changed in future research, more closely described in section 7 .

Of course, while one Dota 2 game takes about 40 minutes to 1 hou $\mathrm{r}$ on average, the typical Hearthstone game lasts for around 5-10 minutes. This is needed to be taken into account, since it is unlikely to preserve above-average HR for a long time during the game.

There are no obvious pattern \$ isible from such small amount of experiments (compared to Dota 2), most likely caused by short and static nature of Hearthstone games. The results seem chaotic at first, but we are confident that after obtaining bigger dataset, some interesting patterns will be uncovered.

After analyzing the patterns we found in our experiments we can put them into two categories:
1. Game-specific patterns: As stated above in this section, there are regular spikes in subject's HR during game of Dota 2.

2. Psychophysiological state patterns: Even though there are several factors affecting psychophysiological state of subject (decribed in 6), we have found interesting readings dependent on subject's current physical state. For example our experiments have shown fairly different HR values depending on time of the day. During the night (00:00 a.m. - 00:04 a.m.), subject was averaging up to 20 less HR than during the day. Surprisingly, the subject did not show much variations in HR after being emotionally involved during the game (e.g. viblent confrontation with teammates/opponents).

\section{CONCLUSIONS, FUTURE WORK}

It has to be taken into consideration, that the domain has a major impact on the final data (even though we try to find a correlation). The data from both games are not easy to compare due to major difference in the game genre and lengths.

After only a few Dota 2 games played by our subject, there are regular alteration in subject's HR. There are specific events which changed the HR whenever they occurred: start ot the game, first massfight, final (deciding) massfight. Also, the subject's HR decreased near to a minimum when waiting for their hero to respawn.

So far there are no particular HR-changing events found in Hearthstone. Nevertheless, this is likely to change after a higher number of experiments, even thought the game is less dynamic than Dota 2.

This paper is a work in progress. We plan to conduct an experiments with multiple subjects in order to identify correlations between user experience and changes in psychophysiological states. We are also aiming to find any reliable correlations between individual psychophysiological attributes (such as [2]). In future, we are also planning to run our experiments on one other genre: First Person Shooter (FPS) games, arguably the most dynamic genre of video-games. Investigating players during a longer session might be useful for determining patterns especially in Hearthstone, since the more-static nature of the game is not showing many variations in HR so far - 6.2. In arena, players are provided 3 lives to win as many games as possible (up to 12) to obtain rewards. In this mode, the players are becoming more engaged in the game and should show interesting HR readings. And finally, we are hoping to find differences between psychophysiological measurements of winning and losing players while understanding its effects on individual subjects.

\section{ACKNOWLEDGEMENT}

Research supported by the National Research and Development Project Grant 1/0773/16 20162019 Cloud Based Artificial Intelligence for Intelligent Robotics.

Received June 15, accepted September 27, 2016 


\section{REFERENCES}

[1] KIVIKANGAS, M. - CHANEL, G. - COWLEY, B. - EKMAN, I. - SALMINEN, M. - JÄRVELÄ, S. RAVAJA, N.: A review of the use of psychophysiological methods in game research, Journal of Gaming \& Virtual Worlds, 2011, 181-199, Intellect.

[2] DRACHEN, A. - NACKE, L. - YANNANAKIS, G. - PEDERSEN, A.: Correlation between heart rate, electrodermal activity and player experience in firstperson shooter games, Proceedings of the 5th ACM SIGGRAPH Symposium on Video Games, 2010, 49-54, ACM.

[3] What Happens When Video Games Can Read Your Face [online], Available at: https://goo.gl/ 7Zt jMH> Accessed: 2016-03-15.

[4] HENDRIKX, M. - MEIJER, S. - VAN DER VELDEN, J. - IOSUP, A.: Procedural content generation for games: A survey, ACM Transactions on Multimedia Computing, Communicatioñs, and Âpplicativins (TOMM), 2013, ACM.

[5] BALLARD, M. - WIEST, J.: Mortal Kombat (tm): The Effects of Violent Videogame Play on Males' Hostility and Cardiovascular Responding1, Journal of Applied Social Psychology, 1996, 717-730, Wiley Online Library.

[6] BARLETT, C. - HARRIS, R. - BRUEY, C.: The effect of the amount of blood in a violent video game on aggression, hostility, and arousal, Journal of Experimental Social Psychology, 2008, 539-546, Elsevier.

[7] CHANEL, G. - REBETEZ, C. - BÉTRANCOURT, M. - THIERRY, P.: Boredom, engagement and anxiety as indicators for adaptation to difficulty in games, Proceedings of the 12th international conference on Entertainment and media in the ubiquitous era, 2008, 13-17, ACM.

[8] DEKKER, A. - CHAMPION, E.: Please biofeed the zombies: enhancing the gameplay and display of a horror game using biofeedback, Proc. of DiGRA, 2007, 550-558.

[9] FLEMING, M. - WOOD, R. - DEBRA, J.: Effects of violent versus nonviolent video games on children's arousal, aggressive mood, and positive mood, Journal of Applied Social Psychology, 2001, 2047-2071, Wiley Online Library.

[10] JARVINEN, A.: Understanding video games as emotional experiences, Video Game Theory Reader 2, 2008, Routledge.

[11] KALLIO, K. - MAYRA, F. - KAIPAINEN, K.: At least nine ways to play: Approaching gamer mentalities, Games and Culture, 2010, 13-17, SAGE Publications.

[12] SCHNEIDER, E.: Death with a Story, Human communication research, 2004, 361-375, Wiley Online Library.

[13] TAFALLA, R.: Gender differences in cardiovascular reactivity and game performance related to sensory modality in violent video game play, Journal of Applied Social Psychology, 2007, 2008-2023, Wiley Online Library.

[14] HAGGMARK, S. - HANEY, M. - JENSEN, S. - JOHANNSON, G. - NASLUND, U.: ST-segment deviations during pacing-induced increased heart rate in patients without coronary artery disease, Clinical Physiology and Functional Imaging, 2005, 246-252, Blackwell Publishing Ltd.

[15] Affectiva [online], Available at: http://http:// www . affectiva. com/Accessed:2016-03-15.

[16] Dota 2 [online], Available at: https://en. wikipedia.org/wiki/Dota_2 Accessed: 201603-15.

[17] Hearthstone: Heroes of Warcraft lonlinel, Available at: https://en.wikipedia.org/wiki/ Hearthstone:_Heroes_of_Warcraft> Accessed: 2016-03-15.

[18] Sample Student Laboratory Report Heart Rate and Cinematic Terror [online], Available at: http://campus.murraystate.edu/academic/ faculty/tderting/samplelab.htm $>$ Accessed: 2016-03-15.

[19] Visualizing HR, HRV, and GSR While Watching Interstellar, Available at: https://goo.gl/JWza4T> Accessed: 2016-03-15.

[20] ZOOK, A.: A Brief History of Matchmaking in Heroes of the Storm [unpublished], nucl.ai Conference 2016, 2016, Available at: https://goo.gl/Ya9YTE> .

\section{BIOGRAPHIES}

Martin Čertický was born on 17.05. 1990. In 2015 he graduated (MSc) at the denartment of Cyhernetics and Artificial Intelligence of the Faculty of Flectrical Encineering and Informatics at Technical University of Košice. He defended his master's thesis in the field of Artificial Intelligence in 2015; his thesis title was "Evolving Reactive Micromanagement Controller for Real-Time Strategy Games". Currently he is a PhD student in the field of Artificial Intelligence. His scientific research is focusing on optimizing user experience in electronic entertainment. In addition, he also investigates questions related with the use of Artificial Intelligence in Video-Games.

Peter Sinčák (born in 1960) is a professor in the branch of artificial intelligence and his main interest is the area of learning systems. Currently he is the head of the Department of Cybernetics and Artificial Intelligence, he is very active in the area of scientific research especially in the area of intelligent robotics. He was a director of IT Valley Košice, in 2009-2012, he was awarded for support of new technologies by Microsoft Slovakia in 2011, he was also awarded by the Mayor of Košice for Internalization of Košice Academic community in 2014. Prof. Sinčák had over 15 invited lectures on leading international universities e.g. Waseda University Tokyo, Japan, Tokyo Institute of Technology, Japan, Chinese University of Hongkong and also in the USA and EU. 